\title{
Linx
}

Revue des linguistes de l'université Paris X Nanterre

$7 \mid 1995$

Saussure aujourd'hui

\section{Saussure et les linguistes russes}

Darius Adamski

\section{OpenEdition}

Journals

Édition électronique

URL : http://journals.openedition.org/linx/1161

DOI : 10.4000/linx.1161

ISSN : 2118-9692

\section{Éditeur}

Presses universitaires de Paris Nanterre

\section{Édition imprimée}

Date de publication : 1 septembre 1995

Pagination : 243-255

ISSN : 0246-8743

\section{Référence électronique}

Darius Adamski, « Saussure et les linguistes russes », Linx [En ligne], 7 | 1995, mis en ligne le 13 juillet 2012, consulté le 20 avril 2019. URL : http://journals.openedition.org/linx/1161 ; DOI : 10.4000/

linx.1161

Ce document a été généré automatiquement le 20 avril 2019.

Département de Sciences du langage, Université Paris Ouest 


\title{
Saussure et les linguistes russes
}

\author{
Darius Adamski
}

« Le style, c'est l'homme ; mais on peut dire que le style c'est, pour le moins, deux hommes, ou, plus exactement, un homme et un groupe social représenté par l'auditeur qui participe de façon permanente au discours intérieur et extérieur de l'homme et incarne l'autorité que le groupe social exerce sur lui. ${ }^{1} »$ Mikhail Bakhtine

« Le style est l'homme même , répète-t-on sans y voir de malice, ni s'inquiéter de ce que l'homme ne soit plus référence si certaine. Au reste l'image du linge parant Buffon en train d'écrire, est là pour soutenir l'inattention. ${ }^{2}$ » Jacques Lacan

Les phrases qui ouvrent ce texte permettent d'emblée d'entrer dans le vif du sujet : là où un Français voit de la malice, un Russe perçoit une autorité ; là où un Français recourt au terme générique d'homme et à un nom de famille, un Russe évoque l'autre, le représentant de la société. Avec Bakhtine, cette attitude peu individualiste donna lieu à un imbroglio : ses écrits auraient été publiés sous le nom de Volochinov ou de Medvedev, ses proches collaborateurs. Notre sujet n'est pourtant pas l'identité de l'auteur des livres qui développent la doctrine du dialogisme; nous traitons de Saussure et de la place qu'il occupe dans la réflexion de Bakhtine, son critique, et dans le structuralisme de ses continuateurs: Nicolas Troubetzkoy et Roman Jakobson. Le nom de Lacan peut surprendre dans ce contexte si l'on oublie que le fondateur de l'Ecole freudienne de Paris intégra les concepts saussuriens dans sa théorie de l'inconscient, qu'il admira le travail de Jakobson, et qu'il fit le plus grand cas de Hegel. Notre thèse est que la Ruse de la Raison de Hegel est le terrain propice à une rencontre entre la malice des Français et l'instinct social des Russes; d'autant plus que, pour la génération de Lacan, l'hégélianisme se confond avec sa version russe fortement épicée de messianisme, celle d'Alexandre 
Kojevnikov, alias Kojève, prophète de la fin de l'Histoire, à la fois philosophe et politicien, qui choisit l'action efficace dans les coulisses plutôt que le devant de la scène. épistémologique, alors que les Russes y voyaient l'exposé final des principes de la grammaire comparée. Il ne nous appartient pas de trancher laquelle des deux interprétations s'accorde davantage avec la réalité des textes ; nous nous contenterons ici d'indiquer le fait que les Français ont reconnu en Saussure le père fondateur, tandis que les Russes ont cherché ses précurseurs. Tel Troubetzkoy qui fait toujours apparaître le nom de Saussure en compagnie de celui de Jan Baudouin de Courtenay, professeur de Kazan et de Saint-Petersbourg que le maître de Genève apprécia et chez qui il puisa ses distinctions entre la langue et la parole, entre la synchronie et la diachronie. Jakobson, quant à lui, repère dans le Cours de linguistique générale « une influence nette de la pensée hégélienne ", dont il trouve "déjà une empreinte incontestable chez Victor Henry " ${ }^{3}$. En effet, dans les Antinomies linguistiques de ce dernier, le mode d'exposition des problèmes est calqué sur le schéma ternaire du mouvement dialectique: au début de chaque chapitre, on trouve une thèse et une antithèse ; à la fin, une synthèse. La synthèse du chapitre final contient la remarque suivante: «Si le langage est un fait conscient, les procédés du langage sont inconscients $»^{4}$.

Dans sa quête des précurseurs, Jakobson remonte jusqu'au Xe siècle et aperçoit la dichotomie langue et parole dans le Dialogue sur l'enseignement de la grammaire de Khrabr, moine bulgare orthodoxe ${ }^{5}$. Pour ce qui est des prédécesseurs immédiats de Saussure, il n'omet jamais l'élève de Baudouin: Mikolaj Kruszewski, qui introduisit dans la linguistique l'associationnisme des philosophes anglais. Les rapports associatifs et syntagmatiques de Saussure correspondent donc aux associations par similarité et par contiguité de Kruszewski. Parmi les sources de celui-ci, Jakobson cite encore Eduard von Hartmann et sa Philosophie de l'Inconscient, « un jalon isolé sur la voie qui mène de Hegel à Husserl $»^{6}$.

3 L'oeuvre de Hartmann, qui popularisa le mot "inconscient ", est composée de deux parties Phénoménologie et Métaphysique. Elle souleva, au moment de sa publication en 1869, une vive polémique. En 1872, Hartmann fit même paraitre anonymement l'Inconscient du point de vue du darwinisme et de la théorie de la descendance où, à l'aide des arguments tenus pour rationnels à l'époque du positivisme triomphant, il réfuta le finalisme de son ouvrage si controversé et si pessimiste. En le rédigeant, il voulut «transformer la philosophie de l'Inconscient, que Hegel professe sans le savoir, en une philosophie consciente d'elle-même $»^{7}$, qui conduirait l'humanité vers sa fin, plus exactement vers l'anéantissement librement consenti par tous. A cette fin, il adapta la pensée hégélienne aux exigences des sciences exactes, et retraça le cheminement de l'Esprit dans l'histoire naturelle: du monde végétal jusqu'à l'homme. Chez l'homme, il détecta l'Inconscient, "l'Un-Tout où l'univers est contenu " ${ }^{8}$, dans la vie végétative du corps, dans l'amour des sexes, dans l'origine du langage et dans la pensée. Le langage est pénétré de l'Inconscient; l'existence des catégories grammaticales universelles en atteste. L'Inconscient est à l'oeuvre dans la pensée; on peut le déduire de l'observation du fonctionnement de l'intuition sensible et des "souvenirs endormis", de ce que Schopenhauer nomma « rumination inconsciente », à savoir cette « intervention féconde 
de l'Inconscient » qu'on saisit mieux dans cette sorte de digestion intellectuelle, à laquelle peut se comparer l'assimilation intérieure des aliments dont la pensée s'est nourrie : c'est que ce travail ne dépend pas de l'impulsion de la conscience, et se produit à un moment qu'on ne saurait déterminer. Nous ne sommes informés qu'il a eu lieu que par les résultats qui le manifestent à l'occasion; mais nous n'avons pas conscience de nous être occupés de la chose. ${ }^{9}$

4 L'Inconscient panthéiste de Hartmann n'équivaut pas à celui de Freud, tout comme la solution finale de la civilisation qu'il suggéra n'est pas celle de Freud qui, tout pessimiste qu'il fût, se contenta de montrer le malaise de vivre avec les formes, comme s'il avait connu la vie sans formes ou, du moins, un tel concept.

Pourtant, dans cet écrit aussi schématique que les caractères nationaux, nous laissons de côté les concepts, en nous en tenant à la lettre, aux mots seuls; et sans négliger leur ambiguïté qui, de tous temps, semble gêner les amateurs des concepts, nous prêtons davantage attention aux évidences banales qu'ils recouvrent, tel un voile. Et question mots, déjà en 1925 dans l'article "En deçà du social », Bakhtine-Volochinov reproche à Freud son subjectivisme hérité de Hartmann, notamment l'importance excessive attachée à la rétrospection individuelle et à la physiologie (le pansexualisme) ${ }^{10}$. Dans le Freudisme paru en 1927, Bakhtine-Volochinov remarque que, pour le père de la psychanalyse, "L'HOMME EST D'ABORD UN ANIMAL », et qu'il ne convient pas de faire l'économie du " second terme de la célèbre formule aristotélicienne : l'homme est un animal SOCIAL " ${ }^{11}$. Après quoi, il démontre que « le rêve, le mythe, la plaisanterie et le mot d'esprit ainsi que toutes les composantes verbales des formations pathologiques $»^{12}$, autrement dit, les réactions verbales que la méthode de libre association met au jour au cours d'une séance analytique, relèvent de l'idéologie du quotidien, celle-ci n'étant pas la propriété d'un individu, mais du groupe social auquel il appartient. Et « toute idéologie est mensongère, tant qu'on n'a pas su y reconnaitre le jeu caché de forces matérielles objectives $»^{13}$.

6 En 1929, dans le Marxisme et la philosophie du langage, Bakhtine-Volochinov critique le formalisme, l'objectivisme abstrait, manifeste dans la distinction nette que Saussure opère entre la langue et la parole. L'objet de la linguistique est la langue, un fait social, alors que la parole, c'est-à-dire l'usage du système de la langue dans les situations concrètes, est totalement individuelle, donc accidentelle, et de ce fait échappe à l'emprise d'une science. Ceci est, selon Bakhtine, le « proton pseudos » de Saussure ${ }^{14}$; qui plus est, la parole étant à l'origine des changements linguistiques, la linguistique saussurienne est dans l'incapacité d'étudier l'histoire de la langue. Pour pallier à ces déficiences, Bakhtine se propose d'élaborer une théorie qui dépasserait l'objectivisme abstrait des Français, voir Saussure, et le subjectivisme des Allemands, voir Freud. Il déclare : "la vérité se trouve au-delà, plus loin, elle manifeste un refus égal de la thèse comme de l'antithèse, et constitue une synthèse dialectique $»^{15}$.

7 Cette élégante application de la dialectique hégélienne abou-tit au dialogisme : Bakhtine rejette la définition saussurienne de l'énoncé, ou de la parole, comme « une combinaison absolument libre des formes de langue ", et précise qu' " on a aussi les formes de combinaison de ces formes de langue $»^{16}$. Il ne renonce donc pas à la conception de la langue envisagée comme le "système où tout se tient", mais l'élargit, en y incluant l'acte de parole ou l'énonciation. Il affirme que "la structure de l'énonciation est une structure purement sociale ", et que «le fait de parole individuel est une (...) contradictio in adiecto ${ }^{17}$. D'après lui, la parole est un dialogue, et le dialogue présuppose la participation active du locuteur et du destinataire : Saussure a eu tort en identifiant la parole au monologue proféré devant un 
destinataire passif. Jakobson est du même avis et avance même que le monologue n'est qu'un dérivé du dialogue, puis ajoute : «la théorie interprétant le sujet parlant comme étant le maître, le propriétaire unique de sa parole est trop simpliste ${ }^{18}$. Où est donc le maître? Dans la dialectique hégélienne, il ne peut exister sans son esclave ou, d'après ceux qui se tiennent religieusement à la lettre de Hegel, sans son valet.

Le mot «énonciation » fit couler beaucoup d'encre en France. Cependant, les idées de Bakhtine n'y connurent une large diffusion qu'après les événements de 1968, grâce aux linguistes d'origine bulgare. En fait, ce fut l'article de Jakobson: « les Embrayeurs, les catégories verbales et le verbe russe ", qui attira l'attention sur la deixis ${ }^{19}$, même si Emile Benveniste s'était de bonne heure intéressé à la disparité entre les pronoms personnels de la 3e personne et ceux de la 1ère et de la $2 \mathrm{e}: \mathrm{je}$, tu, qui ne sauraient être analysés sans le locuteur et le destinataire, instances de discours présentes dans chaque acte de parole. En 1946, il expose cette aporie dans « Structure des relations de personne dans le verbe ». Il observe que seuls les grammairiens arabes aperçurent son importance ${ }^{20}$, et n'imagine même pas que, vers 1927, Baudouin de Courtenay nota :

«Seuls les pronoms de la 1ère personne : je, nous, et de la $2 \mathrm{e}: \mathrm{tu}$, vous, sont dotés d'une signification sociale. La 3e personne ressemble au genre neutre des noms: c'est à proprement parler tout le monde extérieur, au-delà du social. Bien que le monde extérieur contienne également des personnes, mais celles-ci se tiennent en dehors de la communication sociale du moment. ${ }^{21} \ll$

9 L'étude des pronoms personnels conduisit Benveniste à la conclusion qui, depuis son énonciation en 1967, perdit toute sa charge émotionnelle: "Je conçois donc deux linguistiques distinctes $»^{22}$, à savoir une qui perpétue l'héritage saussurien et prend pour objet le système de la langue, et l'autre qui explore le contexte de l'énonciation. Pour Benveniste, tout ce qui a trait à l'énonciation, relève de la « subjectivité ». En employant le mot de subjectivité, il le met pourtant entre guillemets : la subjectivité dont il traite «n'est que l'émergence dans l'être d'une propriété fondamentale du langage », et peu importe «qu'on la pose en phénoménologie ou en psychologie ${ }^{23}$. En fait, son énoncé sous-entend l'existence d'une autre subjectivité, indépendante du langage, mais qui, peutêtre, ne saurait se dire. Il se peut qu'elle soit liée au christianisme, plus précisément à Tertullien (c.160-c.220), auteur célèbre de la maxime censée exprimer l'essence de la foi chrétienne, qu'on cherche en vain dans ses écrits échappés au temps: "Credo quia absurdum ». On y trouve, en revanche, la première application théologique des vocables trinitas et persona, ainsi que le premier essai d'éclairer le mystère de Dieu unique et trine à l'aide de sermo, équivalent latin, à côté de ratio et de verbum, du logos des Grecs: «non posse unum atque eumdem videri qui loquitur, et de quo loquitur, et ad quem loquitur $»^{24}$.

Quelle que soit la subjectivité que vise Benveniste, il reste que, en enterrant l'unité de la linguistique, il fait une concession à la réalité discursive, et peut-être même qu'il le regrette. D'autres parleront de l'échec de la méthode saussurienne, de l'irruption du sujet dans l'édifice de la science, d'une béance dans la langue... Et ces formulations ne sont même pas une hyperbole de ce que les linguistes ou les philosophes français ont écrit sur l'énonciation, car il y a une quinzaine d'années à peine - il faut le rappeler, tant cela peut paraître étrange à qui n'a pas connu l'effervescence de cette époque-là -, l'énonciation était une affaire politique. Cette tension dramatique manque aux écrits des Russes qui, pourtant eux aussi, ont été pris dans les rouages de l'histoire. Chez Jakobson, les 
embrayeurs n'ont rien à voir avec la subjectivité; c'est l'intersubjectivité qu'ils instaurent, et ils sont des faits de langue. De même pour Bakhtine qui déclare à propos de la personnalisation linguistique qu' "elle ne saurait jamais être une subjectivisation. La limite, ce n'est pas moi, mais moi en corrélation avec d'autres personnes, c'est-à-dire : moi et l'autre, moi et toi. ${ }^{25}$ «

Le dédoublement de la linguistique qui, en France, se posa d'une façon douloureuse, ne préoccupa outre mesure ni Jakobson ni Bakhtine. C'était tout au plus une question de terminologie que le premier résolut dans la «sémiotique» et le second, dans la «métalinguistique ». Les bakhtiniens français la baptisèrent «translinguistique ». Voici les raisons de leur choix :

«Bakhtine l'appelle métalinguistique. Ce terme étant aujourd'hui réservé pour distinguer le statut hiérarchiquement supérieur d'un langage enfin vrai sur le langage, dit objet , en tant que système de signes, il serait plus juste de choisir le terme de translinguistique. ${ }^{26}{ }$

« Pour m'inspirer, j'ai relu de Saussure, et je dois dire qu'à la seconde lecture, j'ai été moins enthousiaste, et de loin. En gros, je trouve relativement peu de choses valables dans cette masse de vieux bric-à-brac. Même des choses valables sont terriblement abstraites et peu concrètes. Ce qui fait comprendre la direction qu'ont prise ses disciples. En général, ils pérorent sur le système, mais (...) aucun d'eux n'a su décrire le système d'une langue vivante, même pas du français. »

lettre de Troubetzkoy à Jakobson du 17 mai $1932^{27}$

En 1933, Troubetzkoy définit sa démarche dans le Journal de psychologie. Il se démarque de Saussure, tout en rendant hommage à son précurseur: «La phonologie actuelle ne se borne pas à déclarer que les phonèmes sont toujours membres d'un système, elle montre des systèmes phonologiques concrets et met en évi-dence leur structure ». La linguistique est une science à visée universaliste, et à ce titre, elle se doit de chercher les «lois phonologiques valables pour toutes les langues du monde ». Une de ses tâches pressantes est la constitution d'un atlas linguistique du monde, et cela dans le cadre d'une nouvelle discipline : la " géographie linguistique ». Si Saussure a soutenu que le système de la langue ne peut être étudié que dans la synchronie, la diachronie étant le domaine des faits isolés, Troubetzkoy affirme que «l'évolution du système phonologique est à chaque moment dirigée par la tendance vers un but $\aleph^{28}$. A l'appui, ses travaux sur les langues balkaniques : le bulgare, l'albanais et le néo-grec ne sont pas apparentés génétiquement, et pourtant, ils manifestent des ressemblances de structure dues à leur long voisinage. Il postule donc l'existence des « unions de langues » : pour les langues appartenant à une famille, il est possible de remonter jusqu'à leur protolangue commune, alors que les idiomes d'une union ne manifestent aucune parenté génétique, tout en exhibant de saisissantes similitudes ${ }^{29}$. La Caractéristique de l'union linguistique eurasienne que Jakobson publia à Paris en 1931 prouve le bien-fondé du concept de son maître qui, tout comme lui, aimait l'expression "comme par hasard»: les langues de l'union eurasienne ont pour trait distinctif commun la mouillure des consonnes, et elles s'étendent sur le territoire de l'ancien Empire russe ${ }^{30}$. Cette découverte infirme la thèse de l'arbitraire du signe, chère à Saussure. Les études que Jakobson mènera sur l'aphasie et sur le langage enfantin apporteront de nouvelles preuves du caractère téléologique de la langue, de ce "système des systèmes", comme il le constatera en 1962: "Les deux pprincipes de base de Saussure : l'arbitraire du signe et la linéarité du signans, se sont avérés illusoires $»{ }^{31}$ Pour 
lui, ils l'avaient sans doute été depuis toujours, car en 1939, il voyait dans l'arbitraire du signe " l'esprit anti-finaliste de la fin du siècle dernier », « l'hyper-criticisme destructif de l'époque en question », et assurait : «C'est le sens commun, c'est précisément l'idée que nous, sujets parlants, nous faisons volontiers de la langue qui est parfaitement véridique : la langue est en fait un instrument régi et agencé en vue des concepts à exprimer. ${ }^{32}$ »

Troubetzkoy, en proposant son concept téléologique d'union linguistique, s'appuya sur la théologie et le récit biblique de la Tour de Babel pour argumenter que la diversité des langues n'est pas uniquement un châtiment, comme le prétendent les catholiques romains, car le filet constitué des langues qui recouvrent le globe terrestre ressemble à un arc-en-ciel, l'antique signe de l'alliance entre Dieu et les hommes. L'universalisme de Troubetzkoy, enraciné dans la tradition byzantine, ne fut jamais un oecuménisme uniformisant, mais une revendication de la différence nationale dans le domaine du rite et de la culture ${ }^{33}$. Sa recherche linguistique était, d'ailleurs, une partie intégrante de son projet politique : après la révolution de 1917, il s'installa à Sofia où il fonda le mouvement eurasien. Il croyait que le marxisme sévissant dans sa patrie ne tenait pas compte du caractère national russe. L'eurasisme, dont il était une des figures-phares, se donnait pour objectif de construire une idéologie qui pourrait remplacer la doctrine étrangère, et qui serait tout simplement la description des traits inconscients formant le caractère national russe. A long terme, une telle idéologie devait provoquer une prise de conscience : sous l'influence de l'élite dirigeante, «idéocratie », les Russes deviendraient conscients de leur originalité culturelle et abandonneraient de leur propre gré le marxisme, idéologie des Romano-Germains ${ }^{34}$.

e réaliser son rêve, Troubetzkoy conçut la science de la personnologie dont l'objet était la personne : un être humain, une nation ou leur union. Il para la personne ainsi élargie de l'épithète « symphonique ». La théorie dialogique de Bakhtine, elle aussi, s'orne d'une métaphore musicale : elle est "polyphonique». Si la symphonie fait penser à la collaboration harmonieuse des fidèles au sein de l'église byzantine, la polyphonie évoque les chants qu'on entend dans les temples orthodoxes, particulièrement durant la semaine qui précède la Résurrection. Bakhtine fut du reste condamné à cinq ans de camp de concentration, puis assigné à résidence au Kazakhstan, à cause de sa religion. Pour exposer sa théorie polyphonique, il fit appel à Dostoïevski, écrivain qui personnifie le caractère russe et qui avait horreur de tout ce qui venait de l'Europe, synonyme du déclin moral, sinon du diable. Dostoievski fut également le maitre de Troubetzkoy qui, dans les années trente, lui consacra une série de conférences à l'université de Vienne ${ }^{35}$. Les goûts littéraires de Bakhtine et de Troubetzkoy se rejoignent également dans la personne d'Aleksander Blok. Medvedev, critique véhément des formalistes, lui-même accusé de formalisme, déporté et mort, publia les inédits du poète symboliste connu pour les Vers à la belle dame, mais aussi pour les Scythes ou l'éloge des origines asiatiques du peuple russe, et les Douze ou l'interprétation de la révolution des bolcheviks dans l'esprit du christianisme ${ }^{36}$. Troubetzkoy, lui aussi, aima Blok et publia les Douze pour ses frères en exil.

Troubetzkoy, un émigré, semble être un antimarxiste. Bakhtine et ses amis, qui ne quittèrent jamais la Mère Russie, sont des marxistes. Les titres de leurs livres témoignent de leurs convic-tions, pour autant qu'un titre puisse témoigner de quelque chose quand l'emploi de certains mots signe un arrêt de mort. Jakobson, dans sa préface au Marxisme et la philosophie du langage, rappelle les "concessions à la phraséologie de l'époque", «certains dogmes imposés aux auteurs ». Toujours est-il que tous ces auteurs, y compris 
Troubetzkoy et Jakobson, ont cru avoir trouvé une théorie vraie, ou un système, et ils ont été animés du même esprit, Esprit dont l'incarnation ne fut pas un fait unique. Car selon les dogmes que Hegel définit après la critique du formalisme ignorant de la vie, du travail du concept, après l'analyse des déictiques, formes de langue tout autant universelles que particulières: « je », «ici », «maintenant $»^{37}$, cet Esprit vit son golgotha dans l'Histoire. S'il élit certains individus, ou nations, ce n'est que pour pouvoir entrer en scène : comme si ce n'était pas la vie, mais une représentation théâtrale, unique dans son genre, dont la fin serait sa révélation, en grec apocalypsis.

17 «Il n'y a pas de métalangage », car « Moi, la vérité, je parle... » consigna Lacan fort de sa malice. Mais dans le même écrit: "la Science et la vérité ", il ne manqua pas de mentionner "la ruse consciente des auteurs $»^{38}$. Il fut encore plus explicite dans "Subversion du sujet et dialectique du désir dans l'inconscient freudien » :

« La ruse de la raison veut dire que le sujet dès l'origine et jusqu'au bout sait ce qu'il

veut.

C'est là que Freud rouvre à la mobilité d'où sortent les révolutions, le joint entre vérité et savoir.

En ceci que le désir s'y noue au désir de l'Autre, mais qu'en cette boucle git le désir de savoir. ${ }^{39}$ "

Quant au désir de Lacan, nous citons la dédicace de sa thèse consacrée à La psychose paranoiaque dans ses rapports avec la personnalité : «à mon frère en religion le R.P. MarcFrançois Lacan Moine bénédictin de la Congrégation de France $»^{40}$.$$
* * *
$$

«Je les étonne toujours en leur affirmant que j'ai connu le bonheur, le vrai, l'authentique, la pièce d'or inaltérable qu'on peut échanger contre une poignée de gros sous ou contre une liasse de marks d'après-guerre, mais qui n'en demeure pas moins semblable à elle-même, et qu'aucune dévaluation n'atteint. Le souvenir d'un tel état de choses guérit de la philosophie allemande; il aide à simplifier la vie, et aussi son contraire. »
\end{abstract}

Ce fragment est emprunté à Marguerite Yourcenar et à son Coup de grâce ${ }^{41}$, monologue sur les destinées humaines qui se sont enchevêtrées dans l'horreur de la guerre civile au lendemain de la révolution des bolcheviks, de " ceux qui veulent plus », de ceux qui ont été « majoritaires ", à une voix près. L'action de ce récit français se déroule dans les pays baltes, et à son origine, il y a une histoire que le fils de Ferdinand de Saussure a contée à la romancière ${ }^{42}$.

En 1880, Saussure séjourna en Lithuanie. Ce qu'il y fit, personne ne le sait. Comme il devint par la suite le premier à avoir éclairé le mystère de l'accentuation du lithuanien, selon toute vraisemblance, il avait passé le plus beau de son temps à étudier cet idiome, le plus proche - parait-il - de l'indo-européen, langue mère mythique. Il est néanmoins probable qu'au cours de son séjour, il a rencontré des gens. Je n'insinue pas qu'il ait rencontré le bonheur. Le seul fait qui me semble digne d'être retenu, c'est l'existence de la communauté d'esprit qui se passe d'étiquettes, telles que l'hégélianisme, le marxisme, le freudisme, l'eurasisme, le structuralisme ou le dialogisme qui évoque - ne serait-ce que 
par son nom - les dialogues de Platon, maître de dialectique vendu aux marchands d'esclaves après avoir essayé d'imposer son idéal d'état en Sicile. La communauté dont je signale l'existence n'est pas nécessairement pénétrée de l'esprit de chapelle. Car les beaux esprits ne s'assemblent pas. Leurs chemins se croisent. C'est une question de déplacement :

« Le style c'est l'homme . Le style c'est l'homme même. La première expression est pauvre par sa briéveté épigrammatique. La seconde, correcte, ouvre une toute autre perspective. Elle dit que le style est l'image de l'homme..$^{43}$ »

\section{NOTES}

1. Mikhaïl Bakhtine (V.N.Volochinov), « le Discours dans la vie et le discours dans la poésie » in Tzvetan Todorov, Mikhail Bakhtine le principe dialogique suivi de Ecrits du Cercle de Bakhtine, Seuil, Paris 1981, p. 181-216; la citation p. 212.

2. Ecrits, Seuil, Paris 1966, p. 9.

3. "La théorie saussurienne en rétrospection " in Selected Writings, VIII, Mouton de Gruyer, Berlin, New York, Amsterdam 1988, p. 393-435 ; la citation p. 421. Ce sont de toute évidence les notes $\mathrm{du}$ « Cours sur la doctrine linguistique de Ferdinand de Saussure » que mentionne la notice explicative des Six leçons sur le son et le sens parues au Editions de Minuit en 1976 avec la préface élogieuse de Claude Lévi-Strauss. Si nos déductions sont justes, Jakobson professa son cours en l'année scolaire 1942-1943 à l'Ecole libre des hautes études à New York.

4. Antinomies linguistiques, Ancienne Librairie Germer Baillière et Cie, Félix Alcan - Editeur, Paris 1896 (reprint chez Didier Erudition, Paris 1988), p. 78.

5. «One cf the Speculative Anticipation » in Selected Writings, II, Mouton, the Hague, Paris 1971, p. 369-374.

6. «Relations entre la science du langage et les autres sciences » inEssais de linguistique générale, Minuit, Paris 1973, t.II, p. 9-76; la citation p. 14. Mis à part cet article dédié à Lévi-Strauss, le même volume contient plusieurs écrits rassemblés sous l'intitulé « Précurseurs de la linguistique d'aujourd'hui ».

7. Philosophie de l'Inconscient, Librairie Germer Baillière et Cie, Paris 1877, t.I, p. 31.

8. ibid. t.I, p. 4.

9. ibid. t.I, p. 354 .

10. Ecrits sur le freudisme, l'Age d'homme, Lausanne 1980, p. 37 et s.. Il est à signaler que le titre original « En deçà du social » fut rendu par « Au-delà du social ».

11. ibid. p. 89.

12. ibid. p. 185-186.

13. ibid. p. 172-173.

14. Le Marxisme et la philosophie du langage, essai d'application de la méthode sociologique en linguistique , Minuit, Paris 1977, p. 92.

15. ibid. p. 118.

16. Esthétique de la création verbale, Gallimard, Paris 1984, p. 287.

17. Le Marxisme et la philosophie du langage, p. 141.

18. «La théorie saussurienne en rétrospection », p. 411.

19. Essais de linguistique générale, t.I, Minuit, Paris 1981, p. 176-196 (édition originale 1963). 
20. Problèmes de linguistique générale, t.I, Gallimard, Paris 1966, p. 228.

21. «Rdzenie (pierwiastki, radices), czyli morfemy semantyczne centralne myslenia jezykowego polskiego " (Les racines, ou les morphèmes sémantiques centraux de la pensée langagière polonaise) in O jezyku polskim (De la langue polonaise), PWN, Warszawa 1984, p. 309-322; la citation p. 318-319.

22. « La forme et le sens dans le langage » in Problèmes de linguistique générale, t.II, Gallimard, Paris 1974, p. 215-238; la citation p. 235.

23. «De la subjectivité dans le langage » in Problèmes de linguistique générale, t.I, p. 258-266; la citation p. 260.

24. Tertullien, Liber Adversus Praxeam, cap.XI.

25. Esthétique de la création verbale, p. 391.

26. Julia Kristeva, « une Poétique ruinée » in M. Bakhtine, la Poétique de Dostoïevski, Seuil, Paris 1970 , p. 5-27; la citation p. 13.

27. Troubetzkoy's Letters and Notes, Mouton, the Hague, Paris 1975.

28. « la Phonologie actuelle» in Essais sur le langage, Minuit, Paris 1969, p. 143-164.

29. «Vavilonskaïa bachnia i smechanie ïazykov » (la Tour de Babel et la confusion des langues) in Evraziskiï Vremennik, n 3, Berlin, p. 107-124.

30. «K kharakteristikie evraziïskogo ïazykogo soïuza » in Selected Writings, I, Mouton \& Co., 'SGravenhague 1962, p. 144-201.

31. «Efforts Towards A Means-Ends Model Of Language In Interwar Continental Linguistics » in Selected Writings, II, p. 522-526; la citation p. 524-525.

32. "Les lois phoniques du langage enfantin et leur place dans la phonologie générale " in N.S.Troubetzkoy, Principes de phonologie, Klincksieck, Paris 1986, p. 367-379; la citation p. 379.

33. «Soblazny ïedinienia " (Tentations de l'unification) in Rossiïa i Latinstvo (la Russie et la Latinité), Evraziïskoïe knigoïzdatelstvo, Berlin 1923, p. 121-140.

34. On trouvera un aperçu de l'eurasisme dans K problemie rousskogo samopoznania (Au sujet de la connaissance de soi russe), Evraziïskoïe knigoïzdatelstvo, Pariz 1927. Ses fondements conceptuels sont exposés dans Evropa $i$ celoviecestvo (l'Europe et l'humanité), Rossiïsko-bolgarskoïe Izdatelstvo, Sofia 1920.

35. « O metodakh izuceniïa Dostoïevskogo » (Méthodes pour étudier Dostoïevski) in Novyï zurnal The New Review, n 48, New York 1957, p. 109-121; «O dvukh romanakh Dostoïevskogo » (Deux romans de Dostoïevski), id., n 60, 1960, p. 116-137 ; «Ranniï Dostoïevski » (Le jeune Dostoïevski), id., n 61, 1960, p. 124-146 ; «O vtorom periode tvorcestva Dostoïevskogo » (La seconde période de l'oeuvre de Dostoïevski), id., n 71, 1963, p. 101-127.

36. Pavel N. Medvedev est auteur de Formalnyï metod $v$ literaturovedenii (la Méthode formelle dans les études littéraires), Priboï, Leningrad 1928. Il édita les livres suivants : Pamiati A.A.Bloka (A la mémoire d'A.A.Blok), Polarnaïa zviezda, Peterbourg 1922; Dnevnik Al.Bloka (Journal d'A.Blok), Pisatel, Leningrad 1928 ; Zapisnyïe knizki Al.Bloka(Carnets de notes d'A.Blok), Priboï, Leningrad 1930.

37. Phénoménologie de l'Esprit, Aubier, Paris 1991, la préface et le chapitre premier.

38. Ecrits, p. 855-877, les citations p. 867 et p. 875.

39. Ecrits, p. 793-827, la citation p. 802.

40. De la psychose paranoïaque dans ses rapports avec la personnalité, Le François, Paris 1932. La seconde édition de 1975 au Seuil, qui touche un public plus large du fait de la notoriété de son auteur, corrige la dédicace en : «A mon frère le R.P. Marc-François Lacan, bénédictin de la Congrégation de France ».

41. Alexis ou le Traité du Vain Combat suivi de le Coup de Grâce, Gallimard, Paris 1971, p. 144.

42. Josyane Savigneau, Marguerite Yourcenar, Gallimard, Paris 1990, p. 134.

43. Ludwig Wittgenstein, Remarques mêlées, Trans-Europ-Repress, Mauvezin 1984, p. 93. 